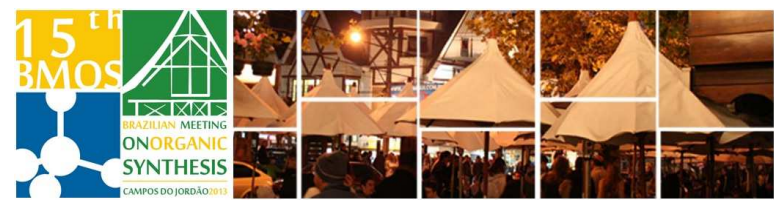

\title{
Organic acids as efficient catalysts for microwave-assisted synthesis of xanthenones
}

\author{
Bruna Silva Terra and Ângelo de Fátima \\ Grupo de Estudos em Química Orgânica e Biológica (GEQOB), Departamento de Química, ICEx, \\ Universidade Federal de Minas Gerais, Belo Horizonte, MG, Brazil. \\ ${ }^{*}$ E-mail corresponding author: brunaterra05@yahoo.com.br
}

Keywords: xanthenones, organic acids, organocatalysis

\section{INTRODUCTION}

Organocatalysis is a process in which metal-free organic molecules are used to accelerate the rate of chemical reactions. ${ }^{1}$ The use of organocatalysts has also been increased due to their low toxicity to the environmental and low sensitivity to oxygen. Natural organic acids (NOA) are biodegradable and thus considered eco- and user-friendly alternatives to synthetic organocatalysts. ${ }^{2}$ This work aimed to evaluate oxalic, malonic, succinic and acetic acids as potential organocatalysts for microwave-assisted synthesis of xanthenones under solvent-free conditions.

\section{RESULTS AND DISCUSSION}

The efficiency of oxalic, malonic, succinic and acetic acids as catalysts under solvent free and microwave irradiation (MW) was first determined using the reaction described in Table 1.

Table 1. Efficiency of natural organic acids (NOA) for the synthesis of xanthenone $4^{\star, \star \star}$.

\begin{tabular}{|c|c|c|c|c|c|}
\hline & benzalde & 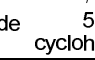 & $\begin{array}{l}\text { methyl } \\
\mathrm{e}-1,3 \text {-dione }\end{array}$ & 4 & \\
\hline \multirow{2}{*}{$\begin{array}{c}\mathrm{NOA} \\
(\mathrm{mol} \%)\end{array}$} & \multirow{2}{*}{$\begin{array}{l}\mathrm{MW} \\
(\mathrm{min})\end{array}$} & \multicolumn{4}{|c|}{ Reaction yield (\%) } \\
\hline & & $\begin{array}{c}\text { Oxalic } \\
\text { acid }\end{array}$ & $\begin{array}{c}\text { Malonic } \\
\text { acid }\end{array}$ & $\begin{array}{l}\text { Succinic } \\
\text { acid }\end{array}$ & $\begin{array}{c}\text { Acetic } \\
\text { acid }\end{array}$ \\
\hline 5 & 5 & 58 & 9 & 10 & 1 \\
\hline 5 & 10 & 51 & 35 & 26 & 58 \\
\hline 10 & 5 & 62 & 16 & 2 & 2 \\
\hline 10 & 10 & 52 & 21 & 51 & 51 \\
\hline 20 & 5 & 82 & 17 & 14 & 51 \\
\hline 20 & 10 & 65 & 54 & 55 & 67 \\
\hline
\end{tabular}

${ }^{*}$ Reagents and conditions: benzaldehyde/ $\beta$-naphtol/5,5-dimethyl1,3-cyclohexanedione (molar ratio $=1: 1.2: 1.5$ ). ${ }^{* *}$ Reactions free of NOA furnished 4 in $16 \%$ and $20 \%$ after 5 and $10 \mathrm{~min}$ of MW, respectively. MW: microwave irradiation.

The best yield reaction was achieved when using 20 mol\% oxalic acid and 5 min of MW. This condition was further used to obtain a series of xanthenones by varying the aldehyde used (Table 2). A wide range of aromatic aldehydes bearing both electron-donor and electron-withdrawing substituents could successfully be used to provide at least 20 xanthenones from either $\beta$-naphtol or sesamol (Table 2).
Table 2. Synthesis of xanthenones under oxalic acidcatalysis*.

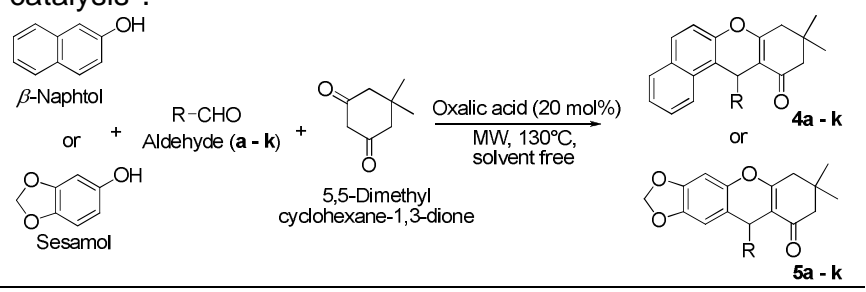

\begin{tabular}{ccc}
\hline & \multicolumn{2}{c}{ Phenolic Compound } \\
\cline { 2 - 3 } Aldehyde (R-CHO) & $\beta$-naftol & Sesamol \\
\cline { 2 - 3 } & Yield (\%) & Yield (\%) \\
\hline $4-\mathrm{NO}_{2}-\mathrm{C}_{6} \mathrm{H}_{4}-(\mathbf{a})$ & $80^{\mathrm{a}}$ & $54^{\mathrm{b}}$ \\
$4-\mathrm{F}-\mathrm{C}_{6} \mathrm{H}_{4^{-}}(\mathbf{b})$ & $72^{\mathrm{a}}$ & $91^{\mathrm{b}}$ \\
$4-\mathrm{Cl}-\mathrm{C}_{6} \mathrm{H}_{4}-(\mathbf{c})$ & $81^{\mathrm{a}}$ & $68^{\mathrm{b}}$ \\
$2-\mathrm{HO}-\mathrm{C}_{6} \mathrm{H}_{4}-(\mathbf{d})$ & $91^{\mathrm{b}}$ & $90^{\mathrm{b}}$ \\
$3-\mathrm{HO}-\mathrm{C}_{6} \mathrm{H}_{4}-(\mathbf{e})$ & $60^{\mathrm{b}}$ & $50^{\mathrm{b}}$ \\
$4-\mathrm{HO}-\mathrm{C}_{6} \mathrm{H}_{4}-(\mathbf{f})$ & $35^{\mathrm{a}}$ & $70^{\mathrm{b}}$ \\
$4-\mathrm{MeS}-\mathrm{C}_{6} \mathrm{H}_{4}-(\mathbf{g})$ & $86^{\mathrm{a}}$ & $79^{\mathrm{b}}$ \\
$4-\mathrm{MeO}-\mathrm{C}_{6} \mathrm{H}_{4}-(\mathbf{h})$ & $70^{\mathrm{a}}$ & $44^{\mathrm{b}}$ \\
$3,4-(\mathrm{MeO})-4-(\mathrm{HO})-\mathrm{C}_{6} \mathrm{H}_{2}-(\mathbf{i})$ & $46^{\mathrm{a}}$ & $62^{\mathrm{a}}$ \\
$3-(\mathrm{MeO})-4-(\mathrm{HO})-\mathrm{C}_{6} \mathrm{H}_{3}-(\mathbf{j})$ & $55^{\mathrm{a}}$ & $63^{\mathrm{a}}$ \\
$\mathrm{C}_{6} \mathrm{H}_{5-}(\mathbf{k})$ & $82^{\mathrm{b}}$ & $67^{\mathrm{a}}$ \\
\hline
\end{tabular}

${ }^{*}$ Reagents and conditions: benzaldehyde/phenolic compound/5,5dimethyl-1,3-cyclohexanedione (molar ratio $=1: 1 \cdot 2: 1.5)$. ${ }^{\mathrm{a}} \mathrm{MW}$ assisted reaction (10 min). ${ }^{b} \mathrm{MW}$-assisted reaction (5 min).

The best time of reaction for obtaining xanthenones from $\beta$-naftol was $10 \mathrm{~min}$ while sesamol furnished better yields from $5 \mathrm{~min}$ reactions regardless the nature of the aldehyde used.

\section{CONCLUSION}

A novel approach was developed for the preparation of xanthenones based on the environmentally friendly use of oxalic acid under MW and solvent-free conditions.

\section{ACKNOWLEDGEMENTS}

This work was supported by FAPEMIG, CAPES and CNPq.

\section{REFERENCES}

(a) Dalko, P. I; Moisan, L. Angew. Chem. Int. Ed., 2004, 43, 5138; (b) Amarante, G. W.; Coelho, F. Quimica. Nova, 2009, 32, 469.

${ }^{2}$ Suresh, A.; Saini, D.; Kumar, J. S.; Sandhu, Green Chemistry Lett. and Rev., 2009, 2, 29. 